\title{
Antimicrobial Activity of Ciprofloxacin-Loaded Softisan-154 Lipid Microparticles: Physicochemical Evaluation and In Vitro Activity
}

\author{
Momoh A Mumuni ${ }^{*}$, Nafiu Aminu ${ }^{2}$, Frankline C Kenechukwu' ${ }^{1}$, Agboke \\ A Ayodeji ${ }^{3}$, Adedokun M Oluseun ${ }^{3}$, Oyeniyi Y James ${ }^{2}$, Parom K Stephen ${ }^{4}$, \\ Obajuluwa A Funke ${ }^{4}$, Usman Muhammad ${ }^{4}$, Darlington Youngson', \\ Tochimen K Michel ${ }^{5}$, Omeje C Ernest ${ }^{6}$ and Emmanuel C Ibezim ${ }^{3}$ \\ ${ }^{1}$ Drug Delivery Research Unit, Department of Pharmaceutics, Faculty of Pharmaceutical \\ Sciences, University of Nigeria Nsukka, Enugu State, Nigeria \\ ${ }^{2}$ Department of Pharmaceutics and Pharm. Microbiology, Faculty of Pharmaceutical \\ Sciences, Usmanu Danfodiyo University, Sokoto, Nigeria \\ ${ }^{3}$ Department of Pharmaceutics and Pharm. Microbiology, Faculty of Pharmacy, \\ University of Uyo, Akwa-Ibom State, Nigeria \\ ${ }^{4}$ Department of Pharmaceutical Microbiology and Biotechnology, Faculty of \\ Pharmaceutical Sciences Kaduna State University, Kaduna, Nigeria \\ ${ }^{5}$ Department of Chemistry, Faculty of Sciences, Usmanu Danfodiyo University, Sokoto, \\ Nigeria \\ ${ }^{6}$ Department of Pharmacognosy and Environmental Medicine Faculty of Pharmaceuti- \\ cal Sciences University of Nigeria Nsukka, Enugu State, Nigeria
}

*Corresponding Author: Momoh A Mumuni, Drug Delivery Research Unit, Department of

Pharmaceutics, Faculty of Pharmaceutical Sciences, University of Nigeria Nsukka, Enugu State, Nigeria.

\begin{abstract}
The objective of this work was to prepare and investigate in vitro ciprofloxacin (CPX)-loaded solid lipid microparticles (SLM) against some selected micro-organisms for improved activity. Lipid matrix consisting of Softisan ${ }^{\circledR}-154$ and Phospholipon ${ }^{\circledR} 90 \mathrm{H}$ was used to prepare SLMs loaded with CPX. The thermal properties of polymers and the SLMs were determined by a differential scanning calorimetry (DSC), particles size and the pH change of CPX-loaded SLMs were evaluated. The encapsulation efficiency (EE), loading capacity (LC) and in vitro release of CPX from the SLMs were evaluated. The antimicrobial activities of the SLM against selected organisms were determined. Characterizations showed that lipid matrix generated imperfect microstructure that accommodated CPX in a concentration-dependent manner. The EE and LC\% were 89.2 and 33\%, respectively. Maximum CPX release of $>80 \%$ was obtained in batch A3. The antimicrobial activity of the formulation against the tested organisms was comparably higher in batch $\mathrm{C}$ $(49.10 \pm 2.10)$ against $S$. aureus and $(33.10 \pm 0.21 \mathrm{~mm})$ against $S$. typhii as compared to the reference sample. This result suggests the capability of the SLMs to modify the release of the drug and improve its activities against the microorganisms.

Keywords: Antimicrobial Activity; Ciprofloxacin; Hybrid; Lipid-Matrix
\end{abstract}

\section{Introduction}

Drug discovery and delivery are among the most challenging aspects of pharmaceutical development and innovations. They involve several trials through carefully navigating the multiples complexities of pharmaceutical sciences and development, keeping in mind the ultimate target of obtaining the required therapeutic goals as well as meeting the regulatory standards [1]. For instance, the pressure on the pharmaceutical industries are on the increase as per the need to develop a new lipids carriers taking the advantage of individual lipids and avoid their disadvantage with the aim of improving it efficacy $[1,2]$. Interestingly, improving drug delivery by tactically engineering the targeting, increase residence time and circulation time, and increase bioavailability among others are the current frontier among players in drug delivery $[3,4]$. The very slow progress in the efficacy of the treatment of severe diseases, coupled with high rate of bacterial resistance to drugs has suggest- ed a growing need for a multidisciplinary approach to the delivery of our commonly used antimicrobial agent to targets in tissues [5]. This has become practically important, because the development of new entity of drug through biotechnology or chemical synthesis are considered very expensive, cumbersome and too many hurdles to cross include the difficulty in getting approvals by regulatory agencies [6]. Ciprofloxacin (CPX) is a synthetic third generation quinolone antibiotic which has the characteristics of being broad spectrum with low side-effects and cross-resistance with other drugs [7]. Currently, CPX exist in several dosage forms: (a). oral, (b). infusion and (c). ophthalmic formulations. However, despite the impressive activities and wide uses of the drug in clinical practice, there are still very huge challenges that need to be surmounted to avoid loss of the useful molecule due to bacteria resistance. The challenges include: poor solubility, low shelf life, toxicity and low plasma concentration of the present conventional tablet, are seri- 
ous challenges that must be resolved. Additionally, CPX because of its bactericidal activity, it is widely prescribed in the treatment of infections cause by gram-negative bacteria [8]. However, efficacy of the drug is greatly affected by its low solubility and poor availability at the site of absorption and these have posed a serious challenge in the clinical utilization of the drug. Therefore, developing sustained release formulation with improved drug solubility, bioavailability and increased residence time at the site of action would be of immense help in reviving and preserving the drug molecule.

In recent times, lipid based drug delivery systems have gained more attention than before among the pharmaceutical formulators due to the arrays of lipids currently available that could be used in different forms for formulations such as solid lipid microparticles (SLMs), solid lipid nanoparticles (SLNs) and microemulsions $[9,10]$. In addition, lipids are promising carriers because of their potential to increase solubility and improve bioavailability of poorly water soluble and/or lipophilic drugs $[11,12]$. It is noteworthy that novel lipid delivery systems such as lipid microparticles and microemulsions can reduce hepatic first pass effect that is associated with conventional oral dosage formulation [13,14]. Asides from protection of the loaded drugs from degradation, SLMs formulations improve mucosal adhesion, residence time in GIT and enhancing sustained release of drug as compared to conventional formulations [14].

The current study is aimed at preparing SLMs consisting of mixture of Phospholipon ${ }^{\circledR} 90 \mathrm{H}$ and Softisan ${ }^{\circledR} 154$ based to enhance the delivery of ciprofloxacin by improving drug encapsulation, controlled release, longer residence time and its antimicrobial activities in vitro. To the best of our knowledge there are no study or report on use of these novel combination of lipid for the delivery of CPX.

\section{Materials and Methods}

Materials

The following materials were used: Phospholipon ${ }^{\circledR} 90 \mathrm{H}(\mathrm{P} 90 \mathrm{H})$ (Phospholipids GmbH, Germany), ciprofloxacin hydrochloride (Juhel Pharmaceuticals Ltd, Nigeria), Softisan ${ }^{\circledR} 154$ (Cremer Oleo GmbH, Hamburg, Germany), sorbic acid, sorbitol (BDH, England), and Polysorbate 80 (Tween ${ }^{\circledR}$ 80) (Merck KGaA, Darmstadt, Germany). All other reagents were used as procured from their manufacturers. Double distilled water (Lion Water, University of Nigeria) was used for SLM preparation.

\section{Preparation of the lipid matrix}

Based on preliminary reports by our group $(15,16)$, lipid matrix consisting of 1:3 of Phospholipon ${ }^{\circledR} 90 \mathrm{H}$ and Softisan ${ }^{\circledR} 154$ was prepared by fusion method. This ratio of P90H and Softisan ${ }^{\circledR} 154$ has been shown to produce nano/microparticles with good drug encapsulation. Briefly, lipids were weighed, melted together on a hot plate at $70^{\circ} \mathrm{C}$ and stirred using a magnetic stirrer (Remi Equip., India), until solidification to get a hybrid lipid matrix.

\section{Formulation of solid lipid microparticles (SLM)}

The fusion or melting method was adopted for the preparation of SLMs. In each case, $10.0 \mathrm{~g}$ of the lipid matrix was weighed, melted at $70^{\circ} \mathrm{C}$ in a temperature-regulated bath until completely melted. Ciprofloxacin (100 mg) was dispersed in the melted lipid and was magnetically stirred. The aqueous phase containing 1.0\% $\mathrm{w} / \mathrm{w}$ Tween ${ }^{\circledR} 80,0.005 \% \mathrm{w} / \mathrm{w}$ thimerosal and $2.0 \% \mathrm{w} / \mathrm{w}$ sorbitol was heated to the same temperature $\left(70^{\circ} \mathrm{C}\right)$ of the melted lipid contain the ciprofloxacin. This aqueous phase was immediately added dropwise to the melted lipid and was homogenized using UltraTurrax homogenizer (T25 basic, Ika Staufen, Germany) at 8000 rpm for $20 \mathrm{~min}$. The dispersion obtained was subjected to probe sonication (AT-500 MW sonicator, India), at $60 \mathrm{~W}$ for $10 \mathrm{~min}$ to obtain CPX-loaded SLMs. The same procedure was used to prepare other batches of SLM with increasing concentrations of ciprofloxacin (250 and $500 \mathrm{mg}$ ) and labeled as A1, A2 and A3. The unloaded or drug free sample was similarly prepared and labeled as A4, making a total of four batches of SLM.

Characterization of the ciprofloxacin-loaded SLMs Determination of percentage recovery of SLMS

The percentage (\%) yield of the SLMs was calculated using the formula:

$\%$ Recovery $=\frac{\mathrm{W}_{1}}{\mathrm{~W}_{2}+\mathrm{W}_{3}} \times 100$---- Eqn. (1)

Where $W_{1}$ is the weight of the SLMs formulated (g), $W_{2}$ the weight of the drug added (g) and $\mathrm{W}_{3}$ the weight of the lipid and other additives (g).

\section{Differential scanning calorimetry (DSC)}

Thermal properties of the lipids matrices and SLMs prepared were evaluated using a differential scanning calorimetry (Shimadzu, DSC, Japan). Briefly, a 3-5.0 mg quantity of each of the formulations was weighed into aluminium pan, hermetically sealed and the thermal behaviour determined in the range, $20-220^{\circ} \mathrm{C}$, under a $10 \mathrm{~mL} / \mathrm{min}$ nitrogen flux at a heating rate of $10^{\circ} \mathrm{C} / \mathrm{min}$ using an empty pan as reference. Similar evaluation was carried out on the individual lipid: Softisan ${ }^{\circledR} 154$, P90H and lipid matrix.

Particle size analysis and morphological characterization of SLMs

The particle sizes of the SLMs were investigated using a polarized light microscopy. In briefly, approximately a drop of the SLMs from each batches was placed on a slide (Marinfield, Germany) using a calibrated glass dropper. It was then covered with a cover slip and viewed under a polarized light microscope (Lieca, Germany) attached with a motic image analyzer (Moticam 2.0, China). With the aid of the software in the microscope, the projected perimeter diameters of the particles corresponding to the particle sizes of the SLMs were determined and averaged. The particle morphologies were also observed and photomicrographs taken. All these were done in a time-dependent manner.

\section{Time-dependent pH stability studies}

The $\mathrm{pH}$ values of SLMs were measured using a digital $\mathrm{pH}$ meter (Horiba, Laqua, Japan). Measurements were made at 24 h, 4 weeks, 8 weeks and 16 weeks. All samples were measured in triplicates and the results were averaged.

Encapsulation efficiency (EE\%) and loading capacity (LC)

Approximately $5.0 \mathrm{ml}$ of the CPX-loaded SLMs was added into a centrifuge tube, and was centrifuged at $4500 \mathrm{rpm}$ at $25^{\circ} \mathrm{C}$ for 50 
min to obtain two phases (i.e. aqueous and lipid phases). A $5 \mathrm{ml}$ volume of the aqueous phase (supernatant) was thereafter diluted 100 -fold using distilled water and filtered (Advantec filter $0.2 \mathrm{mi}-$ cro, Japan). The absorbance of these solutions was determined in a UV-spectrophotometer (Jenway, 605, Germany) at a wavelength of $285 \mathrm{~nm}$, and the encapsulation efficiency (EE) and loading capacity (LC) calculated using equation 2 and 3.

$\mathrm{EE} \%=\frac{\text { Actual drug content }}{\text { Theoretical drug content }} \times 100 .-----$ Eqn.(2)

LC expresses the ratio of the entrapped drug to total weight of the lipids. It is determined as follows:

$\mathrm{LC}=\frac{\mathrm{Pa}-\mathrm{Ps}}{\mathrm{Pa}-\mathrm{Ps}+\mathrm{Pl}} \times 100 .---\mathrm{Eqn}$

Where $P l$ is the initial amount of lipid used in the preparation, $P a$ is the amount of CPX added to the preparation, and Ps is the amount of CPX determined in supernatant after separation of the lipid and aqueous phase.

\section{In vitro release study}

To determine the amount of drug released from the SLMs, dialysis tube technique was used as previously reported [6], with little modification. Briefly, the polycarbonate dialysis membrane used was pre-treated by soaking it in the dissolution medium (phosphate buffer $\mathrm{pH}$ 7.2) for $24 \mathrm{~h}$ prior to the commencement of each release experiment. In each case, $20 \mathrm{mg}$, of each sample was placed in the dialysis bag of length $2 \mathrm{~cm}$, diameter $4 \mathrm{~cm}$, (MWCO 10,000; Spectrum, Los Angeles, USA) containing $2.0 \mathrm{ml}$ of phosphate buffer solution ( $\mathrm{pH} 7.4$ ), securely tied with a thermo-resistant thread and then immersed in the dissolution medium under agitation provided by the magnetic stirrer at $200 \mathrm{rpm}$. At predetermined time intervals, $5 \mathrm{ml}$ of the dissolution medium was withdrawn and replaced with same fresh medium. The amount of CPX in the aliquots was determined at $285 \mathrm{~nm}$ using UV-vis spectrophotometer and the cumulative amount of CPX released from the sample was calculated. The percentage of CPX released was plotted against time. Each data point is mean $( \pm S D)$ calculated from three measurements.

\section{In vitro antimicrobial susceptibility testing}

The antimicrobial activity of the ciprofloxacin-loaded-SLM, conventional tablets (500 mg) and pure sample of ciprofloxacin was tested against two clinical isolates of microorganisms ( $S$. aureus and $S$. typhi) using agar diffusion method previously reported $(6,17)$. The test organisms were standardized using 0.5 MacFarland standards which is equivalent to $1 \times 10^{-8} \mathrm{CFU}$. Therein, Mueller - Hinton agar (MHA) was prepared following the manufacturer's instruction, sterilized at $120^{\circ} \mathrm{C}, 15 \mathrm{psi}$ for $15 \mathrm{~min}$, and allowed to cool to $45^{\circ} \mathrm{C}$. Thereafter, the molten MHA maintained at $45^{\circ} \mathrm{C}$ was aseptically seeded with $0.1 \mathrm{ml}$ of the standardized test organisms and poured into sterile plates. The set up was mixed together and was allowed to gel. Sterile cork borer was used to make holes/wells on the gel agar plate and thereafter $0.1 \mathrm{ml}$ of the test agents (CPXloaded SLM, conventional tablets and pure sample) was added into each well and was allowed to stand for diffusion of the antimicrobial agents. The seeded agar was then incubated for $24 \mathrm{~h}$ at $37^{\circ} \mathrm{C}$. All the tests were done in triplicates and growth was examined after incubation, with the average inhibition zone diameter $(\mathrm{mm})$ of each sample determined.

\section{Stability studies}

Storage stabilities of the sample formulations were evaluated according to the International Conference of Harmonization (ICH) guidelines. The SLMs sample was stored at ambient temperature of $28^{\circ} \mathrm{C}$ for a period of six months, maintained under a spray humidifier (Bottle ORB Model 7098, Topland Co. Japan). At six months, sample was taken, resuspended in distilled water, the particle size, loading capacity and encapsulation efficiency were evaluated.

\section{Statistical analysis}

All data were analyzed using SPSS version 14.0 (SPSS Inc., Chicago, USA) applying one way ANOVA. Mean and standard error for all data were calculated. For batch comparisons, the Student's ttest was used to determine statistically significant differences at $\mathrm{p}<0.05$.

\section{Results and Discussion}

\section{Recovery values}

The recovery values for all the samples of the preparation were generally high and this ranged from 78.6-89.2\%. However, none of the samples achieved $100 \%$ recovery. This could be as result of transference losses during the processing stage. However, the loss could be avoided in an industrial setting where sophisticated equipment is employed.

\section{Thermal properties of the prepared SLM samples}

The DSC thermogram of Softisan ${ }^{\circledR} 154$, Phospholipon ${ }^{\circledR} 90 \mathrm{H}$, lipid matrix and formulated SLMs loaded with increasing concentrations (100, 250 and $500 \mathrm{mg}$ ) of ciprofloxacin were compared (Figure 1). The thermograms of the lipid carrier; $\mathrm{P} 90 \mathrm{H}$ and Softisan ${ }^{\circledR}$ 154 showed sharp endothermic peaks corresponding to melting transitions at $66.5,73.0$ and $61.4^{\circ} \mathrm{C}$ with corresponding enthalpies of $-14.4,-55.57$ and $-41.99 \mathrm{~mW} / \mathrm{mg}$, respectively. Thermograms of the drug-loaded SLMs A1, A2 and A3 showed sharp endothermic peaks at $78.7,75.4$ and $75.9^{\circ} \mathrm{C}$ with corresponding transition enthalpies of $-14.7,-20.66$ and $-15.96 \mathrm{~mW} / \mathrm{mg}$, respectively. The DSC traces of pure ciprofloxacin (Figure 1cpx), had multiple peak with a sharp melting transition at 186.3 with an enthalpy of -12.09 $\mathrm{mW} / \mathrm{mg}$. The thermograph peaks suggest that the drug is completely soluble in the lipid phase. This indicates that the drug (ciprofloxacin) has changed from crystalline state to amorphous state. The changes observed in the melting point and enthalpy of the lipid matrix may be due to structural disorientation of the bond which enhanced drug encapsulation in the lipid material. Similar result was reported (18), where SLM was loaded with an anti-diabetic drug. In this study, it was observed that the thermal behavior of a lipid matrix changed in the presence of other substances such that the melting temperature and changes in enthalpy depended on the nature of interaction between the constituents. The presence of $\mathrm{P} 90 \mathrm{H}^{\circledR}$ reduced the crystallinity of the formulation and this was observed as lower enthalpy and crystallinity index of the lipid matrices. Studies have shown that a decrease in the enthalpy and crystallinity of lipid preparation could possibly cause retention of an entrapped drug over time and may enhance sustained release of the encapsulated drug $[3,19]$. The lower crystallinity may imply imperfect lattice structure which results in pockets of spaces that can accommodate drugs [20]. 


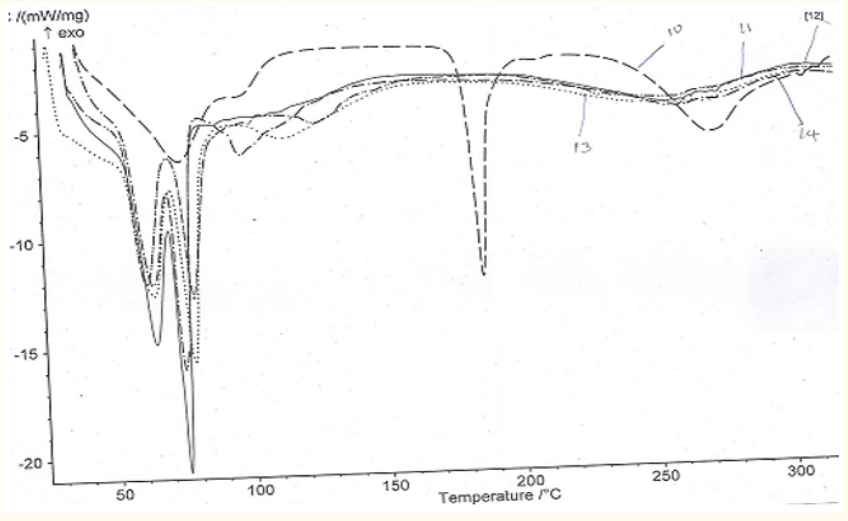

Figure 1: DSC thermograms of SLMs, (10) CPX, (11)

batch A1, (12) batch A2, (13) batch A3, and (14) batch A4.

Morphology and particle size characteristics

The morphology as measured microscopically is shown in Figure 2 and Table 1. The prepared CPX-SLMs were well encapsulated in the lipid matrix, smooth in appearance and spherical in shape.

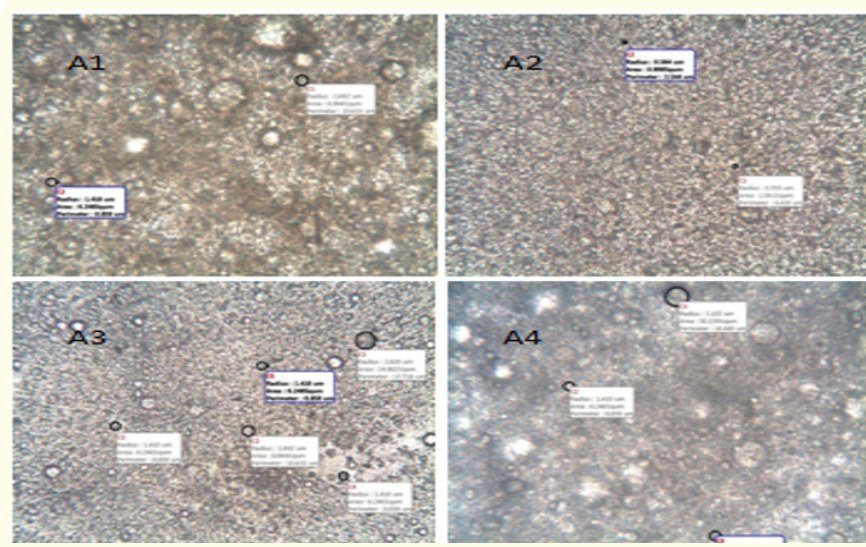

Figure 2: Photomicrographs of batches of SLMs containing

SRMS-154 after 24h of preparation; A1-A3 contain 100, 250 and 500, respectively. Batch A4 contain no CPX.

\begin{tabular}{|l|c|c|c|c|}
\hline & \multicolumn{4}{|c|}{ Particle size $(\boldsymbol{\mu m})$} \\
\hline Batches & $\mathbf{4 8 ~ h}$ & $\mathbf{4}$ weeks & $\mathbf{8}$ weeks & $\mathbf{1 6}$ weeks \\
\hline Al & $62.11 \pm 01$ & $62.02 \pm 21$ & $68.01 \pm 11$ & $75.09 \pm 02$ \\
\hline A2 & $63.11 \pm 00$ & $66.02 \pm 01$ & $70.01 \pm 10$ & $74.09 \pm 10$ \\
\hline A3 & $63.11 \pm 11$ & $70.02 \pm 05$ & $74.01 \pm 21$ & $79.09 \pm 31$ \\
\hline A4 & $59.11 \pm 12$ & $53.02 \pm 11$ & $48.01 \pm 01$ & $41.09 \pm 12$ \\
\hline
\end{tabular}

Table 1: Particle size of CPX-loaded SLMs (mean \pm SD, $n=5$ ).

Key; A1, A2, and A3 mean SLM loaded with 100, 250 and 500 CPX, respectively; While, A4 is unloaded.

\section{pH analysis}

The $\mathrm{pH}$ of the different batches of SLMs was measured $48 \mathrm{~h}, 4$ weeks, and 16 weeks after preparation to ascertain the variation of $\mathrm{pH}$ with time, which could be a function of degradation of the API or excipients or both. Previous reports on lipid formulation have shown changes in their $\mathrm{pH}$ (increase or decrease) which may affect the stability of the preparation as well as therapeutic efficacy $[6,21]$. Thus, this evaluation enables the scientists to monitor the $\mathrm{pH}$ and possible identify cause of changes and provide solution by way of selection of lipid polymer or addition of stabilizer as the case maybe. Figure 3 shows the pH values of the various batches of the SLM formulations. The pH varied from between $6.33 \pm 0.05$ and $7.10 \pm 0.33$ within $24 \mathrm{~h}$ of preparation to between $4.26 \pm 0.12$ and $4.33 \pm 0.21$ within 4 month of preparation, with SLMs containing $500 \mathrm{mg}$ of CPX (C) having the highest reduction in $\mathrm{pH}$ after 16 weeks ( $\mathrm{pH}$ change from $7.10 \pm 0.33$ to $4.33 \pm 0.21$ ). As shown in figure 3 , it is clear that there was a gradual decrease in $\mathrm{pH}$ value of CPX-loaded SLMs as well as the unloaded SLM (A4). The decrease in $\mathrm{pH}$ of the SLMs could be attributed to the degradation of the fatty component into free fatty acid, not of the drug as the changes in $\mathrm{pH}$ also occurred in the unloaded SLM (Drug free batch) This finding is in an agreement with an earlier report $[3,4]$. Interestingly, the fall in $\mathrm{pH}$ of our formulation is within recommended value for CPX dispersed in liquid. However, this slight change in $\mathrm{pH}$ of the formulation did not affect the activity of the CPX as shown in the in vitro study after three months of storage.

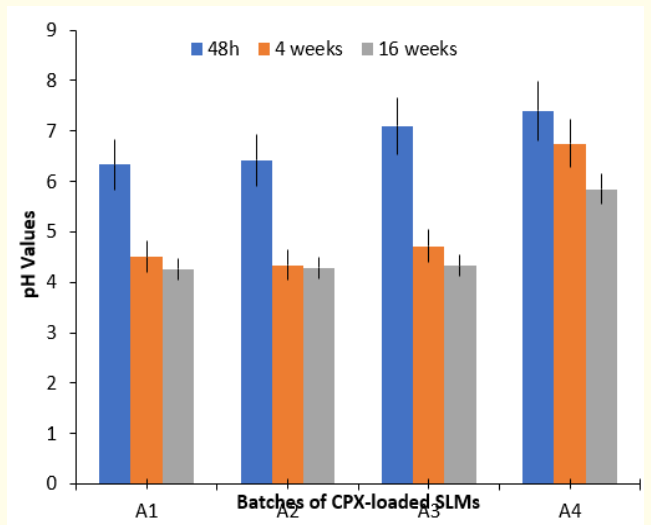

Figure 3: Time resolved $\mathrm{pH}$ analysis of ciprofloxacin-loaded (A1-A3, and unloaded (A4) SLMs.

Key: A1, A2 and A3 contain 100, 250 and 500 of CPX, respectively; while A4 contain no CPX.

\section{Encapsulation efficiency and drug loading capacity}

We reported earlier that the role of the formulated lipid carrier system either in nano/micro is to deliver the drug to the target tissues intact, in higher quantity for effective therapeutic effects. Thus, the capability of the SLM to accommodate active molecules is an important property. It can be expressed by the entrapment efficiency (EE\%) and loading capacity (LC). EE\% defines the ratio between the weights of entrapped drug and the total weight of drug added to the dispersion, while LC expresses the ratio between the entrapped drug and the total weight of the lipids [4]. Figure 4, shows the encapsulation efficiency and drug-loading capacity obtained from the various preparations. The results indicate that the $\mathrm{EE} \%$ and LC increased with increase in the concentration of the drug. High EE\% of model drug was found to be $89.2 \%$ (batch A3 contains $500 \mathrm{mg}$ of $\mathrm{CPX}$ ), indicating that hybrid lipid matrix consisting of $\mathrm{P} 90 \mathrm{H}$ and Softisan-154 promoted concentration-dependent drug encapsulation [19]. Earlier work reported [6] shows that hydrophobic nature of $\mathrm{P} 90 \mathrm{H}$ encourage drug encapsulation in the core of the microparticles. Reports have shown that EE\% depend largely on the lipophilicity of the drug, type of lipid and the ratio in which the lipid is combined as well as the formulation method adopted [4]. 


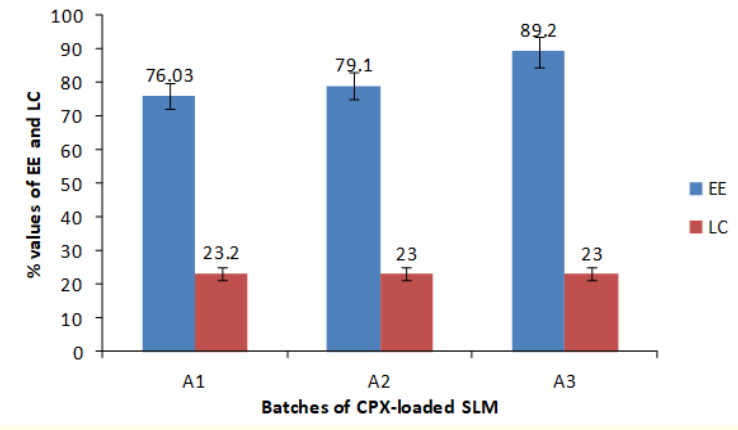

Figure 4: A presentation of encapsulation efficiency (EE) and loading capacity (LC) of prepared SLMs. Key; A1, A2 and A3 mean SLM loaded with 100, 250 and $500 \mathrm{mg}$ of ciprofloxacin respectively.

\section{In vitro ciprofloxacin release study}

Figure 5, shows the in vitro release of the prepared SLMs using the polycarbonate dialysis bag method. Results showed a biphasic release with an initial burst release of $20 \%$ within the first $1 \mathrm{~h}$ and prolonged release over a period of $12 \mathrm{~h}$ in a sustained manner. This effect may be as a result of or could be linked to the desorption and diffusion of un-encapsulated ciprofloxacin at the surface/ peripheral of the particles or weakly bound ciprofloxacin and the partial erosion of the surface of the SLM, thus enhancing the faster diffusion of the drug from the SLM as previously reported [22]. The subsequent release over a period of $12 \mathrm{~h}$ was able to achieve $79 \%$, indicating that the encapsulated drug in the core of the lipid was able to gradually migrate or diffuse into the outer region over time. It was observed that there was no significant difference in the pattern of release among the batches of the formulation. Batch A3 of the formulation showed the highest release of $79 \%$ in $12 \mathrm{~h}$ and the release was sustained for a prolonged time. The release of the CPX from the SLMs was concentration dependent. The sequence of the drug release was $\mathrm{A} 3>\mathrm{A} 2>\mathrm{A} 1$ for 500,250 and $100 \mathrm{mg}$ of $\mathrm{CPX}$ loaded respectively.

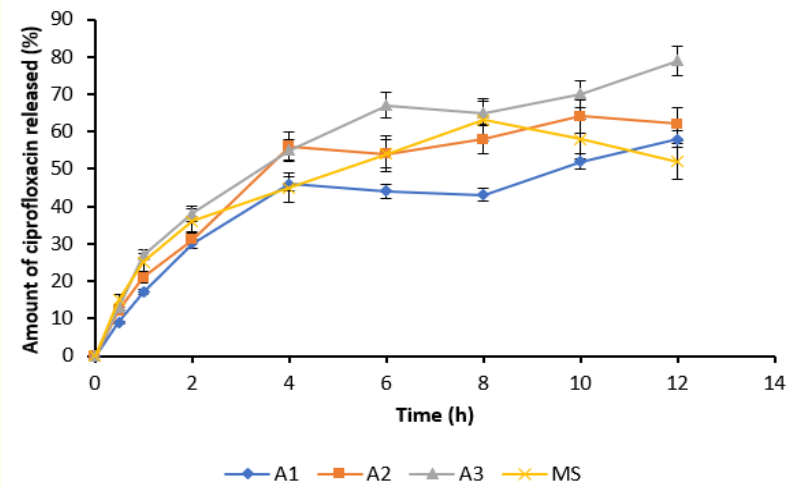

Figure 5: In vitro release profile of ciprofloxacin from SLMs in phosphate buffer at $\mathrm{pH}$ 7.4.

A1, A2 and A3 were formulated with lipid matrices containing 100,250 and $500 \mathrm{mg}$ of CPX, respectively; MS means conventional formulation.
In vitro antimicrobial activity

The antibiotic susceptibility investigations were evaluated using agar dilution method. The zone of activities as a function of inhibitory zone diameter (IZD) is presented in Table 2 and 3. The in vitro antibacterial activities of the formulations were compared with that of pure CPX powder and conventional CPX tablet. The results indicate that CPX loaded SLMs produced very significant ( $p$ $>0.005$ ) zones of inhibition against the tested organisms in a biphasic manner. The early release of un-encapsulated drug or the drug that attached at the surface of the particles shows favorable activities within the first $60 \mathrm{~min}$, with batch A3 showing the highest zone of inhibition ( $34.11 \pm 0.11 \mathrm{~mm}$ ) against $S$. aureus (Table 3), which increased up to $49.10 \pm 2.10 \mathrm{~mm}$ for over $420 \mathrm{~min}$. It appears from the in vitro study that batch A3 showed very high activities as compared to other batches (A1 and A2) of the formulation. Maximum inhibition zone diameter of $49.10 \pm 2.10 \mathrm{~mm}$ was obtained as compared to $39.10 \pm 1.11 \mathrm{~mm}$ and $42.10 \pm 0.22 \mathrm{~mm}$ for batches A1 and A2, respectively. Similar results were observed when the SLMs were tested against $S$. typhi as shown Table 3 . Batch A3 showed inhibition zone diameter (IZD) of $33.10 \pm 0.21 \mathrm{~mm}$ as compared to A1 and A2 with IZDs of $24.30 \pm 0.12$ and $26.40 \pm 2.04 \mathrm{~mm}$, respectively.

\begin{tabular}{|l|c|c|c|c|}
\hline & \multicolumn{4}{|c|}{ Particle size $(\boldsymbol{\mu m})$} \\
\hline Batches & $\mathbf{4 8 ~ h}$ & $\mathbf{4}$ weeks & $\mathbf{8}$ weeks & $\mathbf{1 6}$ weeks \\
\hline $\mathrm{Al}$ & $62.11 \pm 01$ & $62.02 \pm 21$ & $68.01 \pm 11$ & $75.09 \pm 02$ \\
\hline $\mathrm{A} 2$ & $63.11 \pm 00$ & $66.02 \pm 01$ & $70.01 \pm 10$ & $74.09 \pm 10$ \\
\hline $\mathrm{A} 3$ & $63.11 \pm 11$ & $70.02 \pm 05$ & $74.01 \pm 21$ & $79.09 \pm 31$ \\
\hline A4 & $59.11 \pm 12$ & $53.02 \pm 11$ & $48.01 \pm 01$ & $41.09 \pm 12$ \\
\hline
\end{tabular}

Table 1: Particle size of CPX-loaded SLMs (mean \pm SD, $n=5$ ).

Key; A1, A2, and A3 mean SLM loaded with 100, 250 and 500 CPX, respectively; While, A4 is unloaded.

\begin{tabular}{|l|c|c|c|c|c|}
\hline & \multicolumn{5}{|c|}{ IZD (mm) ${ }^{\mathbf{x y}}$} \\
\hline & \multicolumn{5}{|c|}{ Time (min) } \\
\hline Batches & $\mathbf{6 0}$ & $\mathbf{1 2 0}$ & $\mathbf{2 4 0}$ & $\mathbf{3 6 0}$ & $\mathbf{4 2 0}$ \\
\hline \multirow{2}{*}{ A1 } & $27.00 \pm$ & $28.80 \pm$ & $32.10 \pm$ & $34.70 \pm$ & $39.10 \pm$ \\
& 0.13 & 1.10 & 2.11 & 0.19 & 1.11 \\
\hline \multirow{2}{*}{ A2 } & $29.10 \pm$ & $31.70 \pm$ & $35.20 \pm$ & $38.10 \pm$ & $42.10 \pm$ \\
& 0.19 & 0.02 & 0.18 & 0.16 & 0.22 \\
\hline \multirow{2}{*}{ A3 } & $34.11 \pm$ & $37.70 \pm$ & $42.70 \pm$ & $47.22 \pm$ & $49.10 \pm$ \\
& 0.11 & 0.19 & 0.12 & 2.00 & 2.10 \\
\hline \multirow{2}{*}{ A4 } & $00.00 \pm$ & $00.00 \pm$ & $00.00 \pm$ & $00.00 \pm$ & $00.00 \pm$ \\
& 0.00 & 0.00 & 0.00 & 0.00 & 0.00 \\
\hline \multirow{2}{*}{ PC } & $20.13 \pm$ & $22.00 \pm$ & $22.90 \pm$ & $29.10 \pm$ & $32.70 \pm$ \\
& 0.12 & 0.12 & 0.11 & 0.33 & 0.23 \\
\hline \multirow{2}{*}{ MS } & $17.23 \pm$ & $27.98 \pm$ & $33.10 \pm$ & $34.70 \pm$ & $37.70 \pm$ \\
& 0.11 & 0.23 & 0.17 & 0.00 & 0.15 \\
\hline
\end{tabular}

Table 2: Antimicrobial susceptibility testing of formulation against $S$. aureus.

Mean $\pm S D,{ }^{y} n=3$; A-C are SLMs containing 100, 250 and 500 mg of ciprofloxacin, respectively; A4 containing no drug. PC and MS are plain ciprofloxacin and market sample respectively. 
Most strikingly, batch A3 showed significant higher inhibition zone diameter in an in vitro study against both microorganism $(S$. aureus and $S$. typhi) when compared with an equivalent concentration of the pure sample of ciprofloxacin and conventional tablet of ciprofloxacin (both positive controls). Based on the activities of the formulation, it is noteworthy that the antimicrobial activity of the ciprofloxacin was improved using the combination of lipid as carrier. Reports have it that lipid material when used as drug carrier, could improve the activity of the formulation through; polymer penetration to drug, membrane perturbation and membrane disruption due to transduction [2]. Similar results were reported when chitosan/lipids was used as delivery agent for some antibiotics [23].

\section{Stability testing}

The values for EE\%, LC and PS after six months of storage are $85.12 \pm 01,18.51 \pm 61$ and $82.00 \pm 21$, respectively. These parameters are crucial for a good drug delivery system as they affect the release, pharmacokinetics and pharmacodynamics properties of the formulation. However, the slight changes were not significant ( $p>0.05$ ) enough to affect the stability of the preparation. This is in agreement with an earlier report [6], indicates, the stability of the SLM within the experimental conditions of this study.

\section{Conclusions}

Formulation of SLMs based on $\mathrm{P} 90 \mathrm{H}^{\circledR}$ and Softisan ${ }^{\circledR}$-154 were successfully used to ciprofloxacin with improved physicochemical properties property and in vitro study. The CPX-loaded SLMs showed satisfactory results with respect to particle size, encapsulation efficiency and in vitro release study. The in vitro experiments revealed that the CPX-loaded SLM retained its activity after the encapsulation and storage for six months. In addition, the in vitro antimicrobial activity of the CPX-loaded SLM was comparatively higher than that of free CPX and conventional tablet of ciprofloxacin with respect to their inhibition zone diameters. The CPX-loaded SLMs shows a promising alternative method of delivering ciprofloxacin meant for oral administration.

\section{Acknowledgements}

The corresponding author Dr. Momoh is grateful to Tertiary Education Trust Fund (TETfund) National Research Fund (NRF) by Government of Nigeria (Grant number TETFUND/DESS/NRF/ STI/13), for funding this research work.

\section{Competing Interest}

The authors declare no conflict of interest.

\section{Bibliography}

1. Alipour M and Suntres ZE. "Liposomal antibiotic formulations for targeting the lungs in the treatment of Pseudomonas aeruginosa". Therapeutic Delivery 5 (2014): 409-427.

2. Friedrich I., et al. "Drug release and permeation studies of nanosuspensions based on solidified reverse micellar solutions (SRMS)". International Journal of Pharmaceutics 305 (2005): 167.
3. Attama AA and Mu“ller-Goymann CC. "A critical study of physically structured lipid matrices composed of a homolipid from Capra hircus and theobroma oil". International Journal of Pharmaceutics 322 (2006): 67-78.

4. Franklin CK., et al. "Surface-modified mucoadhesive microgels as a controlled release system for miconazole nitrate to improve localized treatment of vulvovaginal candidiasis". European Journal of Pharmaceutical Sciences 111 (2018): 358-375.

5. Umeyor CE., et al. "Preparation of novel solid lipid microparticles loaded with gentamicin and its evaluation in vitro and in vivo". Journal of Microencapsulation 29 (2014): 296-307.

6. Attama AA., et al. "Formulation and in vitro evaluation of a PEGylated microscopic lipospheres delivery system for ceftriaxone sodium". Drug Delivery 16 (2009): 448-457.

7. Turel I., et al. "Compounds of antibacterial agent ciprofloxacin and magnesium - Crystal structures and molecular modeling calculations". European Journal of Inorganic Chemistry (2008): 3718-3727.

8. Aristilde L and Sposito G. "Binding of ciprofloxacin by humic substances: a molecular dynamics study". Environmental Toxicology and Chemistry 29 (2010): 90-98.

9. Severino P., et al. "Polymorphism, crystallinity and hydrophilic-lipophilic balance of stearic acid and stearic acid-capric/caprylic triglyceride matrices for production of stable nanoparticles". Colloids Surf B Biointerfaces 86 (2011): 125-130.

10. Xia J., et al. "Nosocomial infection and its molecular mechanisms of antibiotic resistance". BioScience Trends 10 (2016): 14-21.

11. Zhang ZR., et al. "In vivo biodistribution, anti-inflammatory, and hepatoprotective effects of liver targeting dexamethasone acetate loaded nanostructured lipid carrier system". International Journal of Nanomedicine 5 (2010): 487-497.

12. Mbah CC. "Formulation and evaluation of ethosomes for the delivery of four bioactive agents. PhD Thesis". Department of Pharmaceutics University of Nigeria, Nsukka, Nigeria (2013).

13. O'driscoll CM and Griffin BT. "Biopharmaceutical challenges associated with drugs with low aqueous solubility-the potential impact of lipid-based formulations". Advanced Drug Delivery Reviews 60 (2008): 617-624.

14. Shah NV., et al. "Nanostructured lipid carriers for oral bioavailability enhancement of raloxifene: design and in vivo study". Journal of Advanced Research 7 (2016): 423-434.

15. Mumuni AM and Charles OE. "Phospholipon 90H (P90H)based PEGylated microscopic lipospheres delivery system for gentamicin: an antibiotic evaluation". Asian Pacific Journal of Tropical Biomedicine (2012): 889-894. 
16. Kenechukwu FC., et al. "Solid lipid microdispersions (SLMs) based on PEGylated solidified reverse micellar solutions (SRMS): A novel carrier system for gentamicin". Drug Delivery 22 (2015): 710-722.

17. Clinical and Laboratory Standards Institute (CLSI). Reference methods for broth dilution antifungal susceptibility testing of yeast. In: Approved Standard M27-A3, $3^{\text {rd }}$ edition. Clinical and Laboratory Standards Institute (2008).

18. Nnamani PO., et al. "SRMS142-based solid lipid microparticles: Application in oral delivery of glibenclamide to diabetic rats". European Journal of Pharmaceutics and Biopharmaceutics 76 (2012): 68-74.

19. Chukwuma OA., et al. "Formulation, characterization and antimalarial activity of homolipid-based artemether microparticles". International Journal of Pharmaceutics 478 (2015): 202-222.

20. Severino P., et al. "Development and characterization of a cationic lipid nanocarriers as non-viral vector for gene therapy". European Journal of Pharmaceutical Sciences 66C (2014): 7882.

21. Sadeek SA., et al. "Spectroscopic, structure and antimicrobial activity of new Y(III) and Zr(IV) ciprofloxacin". Spectrochimica Acta Part A: Molecular and Biomolecular Spectroscopy 78 (2011): 854-867.

22. Nabanita K., et al. "Development and evaluation of a cedrolloaded nanostructured lipid carrier system for in vitro and in vivo susceptibilities of wild and drug resistant Leishmania donovani amastigotes". European Journal of Pharmaceutical Sciences 104 (2017): 196-211.

23. Govindarajan VK., et al. "Ciprofloxacin loaded genipin crosslinked chitosan/heparin nanoparticles for drug delivery application". Materials Letters 180 (2016): 119-122.

\section{Assets from publication with us}

- Prompt Acknowledgement after receiving the article

- Thorough Double blinded peer review

- Rapid Publication

- Issue of Publication Certificate

- High visibility of your Published work

Website: www.actascientific.com/

Submit Article: www.actascientific.com/submission.php Email us: editor@actascientific.com

Contact us: +919182824667 